\title{
A Phase II Study of Antineoplastons A10 and AS2-1 in Children with Recurrent, Refractory or Progressive Primary Brain Tumors-Final Report (Protocol BT-22)
}

\author{
Stanislaw R. Burzynski, Tomasz J. Janicki, Gregory S. Burzynski, Ania Marszalek, \\ Sheldon Brookman \\ Burzynski Clinic, Houston, Texas, USA \\ Email: gsb@burzynskiclinic.com \\ Received 13 July 2014; revised 8 August 2014; accepted 3 September 2014 \\ Copyright (C) 2014 by authors and Scientific Research Publishing Inc. \\ This work is licensed under the Creative Commons Attribution International License (CC BY). \\ http://creativecommons.org/licenses/by/4.0/

(c) () O) Open Access

\section{Abstract}

Primary malignant brain tumors are a leading cause of cancer-related death in children. This Phase II study evaluated the efficacy and safety of Antineoplastons A10 and AS2-1 (ANP) in children who developed progression during standard treatment. A total of 43 children were recruited to the study, but only 41 met eligibility criteria. There were twelve cases of glioblastoma multiforme (GBM), eight anaplastic astrocytomas (AA), twelve diffuse intrinsic pontine gliomas (DIPG), three supertentorial primitive neuroectodermal tumors (sPNET), three cases of medulloblastoma and one case each of anaplastic ependymoma (AE), atypical teratoid rhabdoid tumor (AT/RT), and disseminated pilocytic astrocytoma (PAD). ANP was administered intravenously daily every four hours (median dose of A10 8.74 g/ kg/d and AS2-1 0.35 g/ kg/d), until objective response (OR) was documented, and then a further eight months. All enrolled patients were included in safety, but only eligible patients in the efficacy evaluation. A total of $12.2 \%$ of patients obtained $0 \mathrm{R} ; 2.4 \%$ complete response (CR) and 9.8\% partial response (PR). Stable disease (SD) was determined in $17.1 \%$ and progressive disease (PD) in $43.9 \%$ of cases. There were $26.8 \%$ of nonevaluable (NE) cases due to premature discontinuation. Out of five OR cases, four patients were diagnosed with recurrent DIPG and one with recurrent AA. Median progression-free survival (PFS) was 2.5 months. Median overall survival was 4.8 months. OS at 6 months was $46.3 \%$, one year was $12.2 \%$, and $4.8 \%$ at two, five, and ten years. The longest survivor is a patient diagnosed with DIPG and gliosarcoma who remains alive more than 15 years. A group of eleven patients reported grade 3 and 4 toxicity including hypernatremia in eight cases, somnolence in two cases, and hypokalemia in one case. There were no chronic toxicities, and the quality of life was very good. The largest group of patients were represented by DIPG, GBM, and AA. The best results were obtained in the DIPG and AA groups. In the DIPG group, CR was in $8.3 \%$, PR was 25\%, median PFS was 4.8 months, 
median OS was 6.1 months, and OS at 6 months was $58.3 \%$, at one year $25 \%$, and $8.3 \%$ at two, five, and ten years. In the AA group, PR was 12.5\%, median PFS was 3.7 months, median OS was 4.7 months, and OS at 6 months was $37.5 \%$, and $12.5 \%$, at one, two, five, and ten years. In conclusion, antineoplastons showed efficacy and acceptable toxicity in patients with recurrent, refractory or progressive primary brain tumors.

\section{Keywords}

\section{Anaplastic Astrocytoma, Antineoplastons A10 and AS2-1, Brainstem Glioma, Diffuse Intrinsic Pontine Glioma (DIPG), Gliosarcoma, Phase II Clinical Trial, Recurrent Glioma}

\section{Introduction}

Pediatric primary malignant brain tumors are a leading cause of cancer-related deaths in children [1]. Pediatric high-grade gliomas (HGG) are relatively uncommon and represent approximately $17 \%$ of all pediatric brain tumors [2]. Supratentorial HGG and diffuse intrinsic pontine glioma (DIPG) continue to be two main challenges for pediatric oncologists [3]. The standard of care for newly diagnosed HGG is surgical resection followed with daily temozolomide (TMZ) and radiation therapy (RT) with subsequent adjuvant TMZ [4] [5]. Standard RT continues to be the main treatment for newly diagnosed DIPG. Unfortunately, there are no standard therapy recommendations after recurrence [5]-[7].

Antineoplastons A10 and AS2-1 (ANP) have been tested extensively in Phase II studies in primary brain tumors. Initial clinical responses in the treatment of HGG and DIPG convinced us to design and conduct a single-arm Phase II study of ANP therapy to assess the efficacy and safety in children diagnosed with primary malignant brain tumors, mostly gliomas, which failed to respond to standard therapy [8]-[12].

\section{Patients and Methods}

\subsection{Patient Population}

Recruited patients were between 6 months old and 18 years with radiologic evidence of recurrent, refractory, progressive or persistent primary brain tumors after receiving standard therapy, by gadolinium-enhanced magnetic resonance imaging (MRI) performed within 14 days prior to initiating the study. Patients were required to have a histologically confirmed, incurable primary malignant brain tumor or DIPG, and to have a complete recovery and at least four weeks from prior surgical procedure. At least eight weeks must have elapsed since the last dose of RT, and at least four weeks must have elapsed since the last dose of chemotherapy (six weeks for nitrosoureas) or immunotherapy. However, patients with clear evidence of disease progression during the initial therapy could be enrolled less than 8 weeks following the last dose of RT, less than 4 weeks after surgery or the last dose of chemotherapy, if the investigator determined that it was safe to administer ANP to such patients.

Diagnosis made by MRI without the necessity of a biopsy is generally accepted for brainstem glioma (BSG). Based on MRI alone, DIPG could be diagnosed if the tumor has an epicenter in the pons and involved more than $50 \%$ of the pons. Patients with tumors that involved the thalamus or upper cervical regions of the spinal cord were also classified as DIPG if the lesion primarily involved the brainstem. Patients with neurofibromatosis were not covered by this definition and were not included. Tumors involving less than $50 \%$ of the pons or exophytic or exophytic involving from pons were classified as DIPG if they had anaplastic, glioblastoma (GBM) or gliosarcoma (GS) histology [6] [13]-[15]. The remaining types of BSG included focal, exophytic, cervicomedullary, and midbrain tumors [16] [17].

Patients were required to have a gadolinium-enhanced MRI performed within two weeks prior to study enrollment. There were no exclusion criteria based on tumor size, multifocality or leptomeningeal or the presence of systemic metastases. Eligibility criteria also included a Karnofsky Performance Status (KPS) of 60 - 100. A group of patients admitted, based on Special Exceptions permitted by the Food and Drug Administration (FDA), had a KPS below 60. The subjects were required to have relatively normal hematopoietic and hepatic function

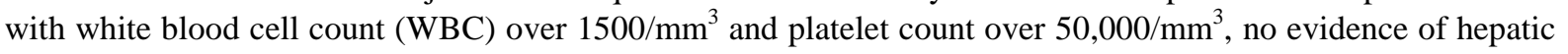


or renal insufficiency, and a total bilirubin and serum creatinine of no higher than $2.5 \mathrm{mg} / \mathrm{dL}$ and SGOT and SGPT no higher than $5 \times$ the upper limits. Use of corticosteroids was permitted to reduce symptoms and signs attributed to cerebral edema, but it was recommended that the smallest doses compatible with the preservation of optimal neurologic function be used. Confirmation of the pathologic diagnosis by an outside pathologist was also required.

The exclusion criteria included serious active infection, fever or other serious concomitant disease that would interfere with the evaluation of the treatment (e.g., severe heart or lung disease). Criteria for the removal from the study were the same as in studies BT-06 and BT-11, and were described previously [10] [12].

All study subjects and/or guardians read, understood, and signed written informed consents prior to enrollment. This study was conducted in accordance with the US Code of Federal Regulations, Title 21, Parts 11, 50, 56, and 312; the Declaration of Helsinki (1964) including all amendments and revisions; the Good Clinical Practices: Consolidated Guideline (E6); International Conference on Harmonization; and the FDA Guidance for Industry. The study was sponsored by the Burzynski Research Institute, Inc., (BRI) and conducted by the Burzynski Clinic (BC) in Houston, Texas.

\subsection{Study Design}

The study was a single-arm, two-stage, interventional Phase II trial of ANP as the monotherapy in a high-risk, poor-prognosis study population. The study was listed by the National Cancer Institute (NCI) under the number of NCT00003476. The study was overseen by an independent Institutional Review Board.

The study was performed according to Protocol BT-22 and conducted under the U.S. IND 43,742. The study commenced on March 6, 1996 and was closed to accrual on November 15, 2011. The protocol was amended by BRI several times; however, none of the amendments altered the aim or design of the original study objectives.

\section{Statistical Consideration}

The sample size and statistical methods were based upon the method described by Chang et al. and were described before [18]. A response rate to ANP of $\geq 10 \%$ was considered 'of interest,' and the primary endpoint was to determine the overall response rate (confirmed complete response (CR) or partial response (PR)) to ANP therapy. An interim analysis would be conducted after 20 subjects had enrolled in the study. If one or more patients achieved a confirmed radiographic response, an additional 20 subjects would be recruited. Overall survival (OS) was measured from the first day of ANP administration until death from any cause, and progression-free survival (PFS) was measured from the first day of the treatment until the date of first observation of progressive disease (PD), beginning of other treatment or death. OS and PFS were estimated by Kaplan-Meier analysis using the MedCalc Statistical Software version 13.3 (MedCalc Software bvba, Ostend, Belgium; 2014). The primary endpoint was the response to treatment which was determined in these different populations: 1) study eligible, 2) DIPG, and 3) anaplastic astrocytoma (AA). Time to best response, dosage, and duration of treatment were analyzed. The average maximum effective daily dose and the range were calculated.

\section{Treatment Plan}

ANPs A10 and AS2-1 were delivered via a dual-channel infusion pump and a single-lumen subclavian catheter (Broviac, Groshong or Hickman) every 4 hours as described before [10] [12]. Criteria for using additional medications including corticosteroids and for outpatient treatment and monitoring were previously described [10] [12].

\section{Evaluation and Follow-Up}

Tumor measurements were based on contrast-enhanced lesions identified by MRI, T2 and FLAIR images were also evaluated [10] [15] [19]. Blood and urine tests and additional measurements were performed as previously described [10] [12]. MRIs were repeated at least every 8 weeks during the first 2 years unless the patient's condition or confirmation of response required an MRI within 4 weeks. Positron emission tomography (PET) scans were performed as necessary. The adverse events were evaluated in all patients and graded according to the Common Terminology Criteria for Adverse Events v. 3 (CTCAE v.3). 


\section{Results}

\subsection{Patient Demographic}

Subject enrollment started March 6, 1996 and continued through November 15, 2011. As of May 15, 2013, all subjects were removed from the therapy due to a CR, PR, subject request, PD, or worsening clinical condition. The results were analyzed in a group of 43 patients from which only 41 were eligible for efficacy. Two patients with AA were found ineligible; none of them received prior treatment. The response of both patients was classified as stable disease (SD). The largest groups were patients with DIPG, GBM, and AA. The best results were obtained in the DIPG and AA groups. The characteristic of the eligible patients are summarized in Table 1.

\section{Table 1. Patient characteristics.}

\begin{tabular}{|c|c|}
\hline Characteristic & Number \\
\hline Age (range years) & $1.4-18.5$ \\
\hline Male & 20 \\
\hline Female & 21 \\
\hline \multicolumn{2}{|l|}{ Race } \\
\hline White & 36 \\
\hline African-American & 1 \\
\hline Latin American & 3 \\
\hline Oriental & 1 \\
\hline \multicolumn{2}{|l|}{ Prior therapy } \\
\hline Surgery and chemotherapy & 3 \\
\hline Surgery and radiation & 3 \\
\hline Chemotherapy only & 1 \\
\hline Radiation only & 5 \\
\hline Chemotherapy and radiation & 5 \\
\hline Surgery, chemotherapy, and radiation & 16 \\
\hline Surgery, chemotherapy, radiation and ABMT/SCR & 4 \\
\hline Surgery, radiation and targeted/biological therapy & 2 \\
\hline Surgery, radiation, chemotherapy and targeted/biological therapy & 1 \\
\hline Radiation and targeted/biological therapy & 1 \\
\hline \multicolumn{2}{|l|}{ Diagnosis } \\
\hline DIPG & 12 \\
\hline GBM & 12 \\
\hline AA & 8 \\
\hline Medulloblastoma & 3 \\
\hline sPNET & 3 \\
\hline $\mathrm{AE}$ & 1 \\
\hline $\mathrm{AT} / \mathrm{RT}$ & 1 \\
\hline PAD & 1 \\
\hline \multicolumn{2}{|l|}{ Karnofsky performance score } \\
\hline Median & 50 \\
\hline Range & $40-90$ \\
\hline
\end{tabular}

Abbreviations: AA—anaplastic astrocytoma; AE—anaplastic ependymoma; AT/RT—atypical teratoid rhabdoid tumor; ABMT—autologous bone marrow transplant; DIPG—diffuse intrinsic brainstem glioma; GBM—glioblastoma multiforme; PAD—disseminated pilocytic astrocytoma; SCR— stem cell rescue; sPNET—-supratentorial primitive neuroectodermal tumor. 
Among 41 eligible patients, there were 12 cases of GBM, 8 anaplastic astrocytoma (AA), 12 DIPG, 3 medulloblastoma, 3 supratentorial primitive neuroectodermal tumor (sPNET), and 1 each of anaplastic ependymoma (AE), atypical teratorhabdoid tumor (AT/RT), and disseminated pilocytic astrocytoma (PAD). Recurrent tumors among patients with objective response (OR) and SD were diagnosed in 92\%, and persistent tumors in $8 \%$.

\subsection{Treatment}

The median daily doses of ANP A10 and AS2-1 are described in Table 2.

The median time to first OR was 19 weeks (range 7 to 38 weeks). The duration of IV ANP therapy ranged from 0.4 to 77 weeks with a median of 13.7 weeks. One subject had a CR and therapy ended after 59 weeks of IV treatment. Six patients halted the treatment based on their decision. Eight subjects halted therapy due to PD confirmed by MRI. In 26 cases, ANP were stopped due to worsening of their clinical condition and 6 patients died while on treatment from the underlying disease.

\section{Responses and Survival}

The data on confirmation of diagnosis, recurrence and response among OR and SD patients are shown in Table 3.

Table 4 shows the response rate and survival for study eligible population as well as for subgroups.

A total of five patients (12.2\%) obtained OR to ANP-one case of CR (2.4\%) and four cases of PR (9.8\%). A patient diagnosed with DIPG and disseminated GS achieved a CR, and is now surviving over 15 years since treatment start (Figure 1) [11].

SD was determined in 7 cases (17.1\%) and PD in 18 cases (43.9\%). There were $11 \mathrm{NE}$ cases (26.8\%) due to premature discontinuation of treatment based on the patient's decision in 3 cases, and deterioration of patients' condition in 8 cases, 2 patients from this group died due to underlying malignancy while on treatment. In the eligible study group, OS was $46.3 \%$ at 6 months, $12.2 \%$ at one year and $4.8 \%$ at two, five and ten years. The PFS at six months was 22\%. In the DIPG group, OS was 58.3\% at 6 months, $25.0 \%$ at one year and $8.3 \%$ at two, five and ten years. The PFS at six months was $41.7 \%$. In the AA group, OS was $37.5 \%$ at 6 months, $12.5 \%$ at one, two, five and ten years. The PFS at six months was $25.0 \%$. The Kaplan-Meier overall survival and progression-free survival curves for all patient cases are presented in Figure 2 and Figure 3. Detailed descriptions of some successfully treated cases have been presented in interim reports [8] [11].

The comparison of the results of treatment with ANP versus temozolomide in recurrent or progressive brain tumors in children are shown in Table 5.

Table 2. Median daily dose of ANP A10 and AS2-1.

\begin{tabular}{|c|c|c|c|c|c|c|c|}
\hline \multirow[b]{3}{*}{$\begin{array}{l}\text { Treated } \\
\text { patients }\end{array}$} & & \multicolumn{6}{|c|}{$\begin{array}{l}\text { Median daily dose } \mathrm{g} / \mathrm{kg} / \mathrm{d} \\
\text { (Range) }\end{array}$} \\
\hline & & \multicolumn{2}{|c|}{ Entire treatment } & \multicolumn{2}{|c|}{ Dose until documented OR achieved } & \multicolumn{2}{|c|}{ Dose until documented SD achieved } \\
\hline & $\mathrm{N}$ & A10 & AS2-1 & A10 & AS2-1 & A10 & AS2-1 \\
\hline All & 41 & $\begin{array}{c}8.74 \\
(0.48-24.58)\end{array}$ & $\begin{array}{c}0.35 \\
(0.13-0.51)\end{array}$ & $\begin{array}{c}10.58 \\
(9.46-16.89)\end{array}$ & $\begin{array}{c}0.35 \\
(0.32-0.44)\end{array}$ & $\begin{array}{c}9.23 \\
(6.75-24.58)\end{array}$ & $\begin{array}{c}0.39 \\
(0.27-0.47)\end{array}$ \\
\hline DIPG & 12 & $\begin{array}{c}10.26 \\
(1.85-24.58)\end{array}$ & $\begin{array}{c}0.37 \\
(0.27-0.51)\end{array}$ & $\begin{array}{c}11.46 \\
(9.46-16.89)\end{array}$ & $\begin{array}{c}0.40 \\
(0.34-0.44)\end{array}$ & $\begin{array}{c}8.65 \\
(6.75-24.58)\end{array}$ & $\begin{array}{c}0.41 \\
(0.27-0.47)\end{array}$ \\
\hline $\mathrm{AA}$ & 8 & $\begin{array}{c}6.29 \\
(1.93-13.09)\end{array}$ & $\begin{array}{c}0.30 \\
(0.22-0.44)\end{array}$ & $9.87^{*}$ & $0.32^{*}$ & $7.88^{*}$ & $0.29^{*}$ \\
\hline GBM & 12 & $\begin{array}{c}8.47 \\
(1.61-13.38)\end{array}$ & $\begin{array}{c}0.33 \\
(0.13-0.46)\end{array}$ & - & - & $\begin{array}{c}12.04 \\
(10.72-13.36)\end{array}$ & $\begin{array}{c}0.40 \\
(0.39-0.40)\end{array}$ \\
\hline
\end{tabular}

* Only one patient. Abbreviations: AA—anaplastic astrocytoma; DIPG—diffuse intrinsic pontine glioma; GBM—glioblastoma multiforme; OR—objective response; $\mathrm{SD}$ - stable disease. 
Table 3. Confirmation of diagnosis, recurrence and response.

\begin{tabular}{|c|c|c|c|c|c|c|c|c|c|c|c|c|}
\hline \multicolumn{5}{|c|}{ Confirmation of diagnosis } & \multirow[b]{4}{*}{ tment } & \multirow{2}{*}{\multicolumn{2}{|c|}{$\begin{array}{l}\text { Confirmation of } \\
\text { recurrence }\end{array}$}} & \multirow{2}{*}{\multicolumn{2}{|c|}{$\begin{array}{c}\text { Confirmation of response } \\
\text { to ANP }\end{array}$}} & \multirow{2}{*}{\multicolumn{3}{|c|}{ Tumor measurements }} \\
\hline & Pathology & & Radiolog & & & & & & & & & \\
\hline \multirow[b]{2}{*}{ : } & \multirow[b]{2}{*}{  } & \multirow[b]{2}{*}{ 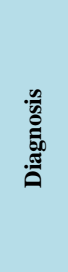 } & \multirow[b]{2}{*}{ 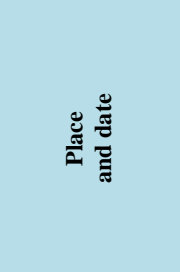 } & \multirow[b]{2}{*}{ 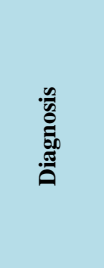 } & & \multirow[b]{2}{*}{ ปั๊ } & \multirow[b]{2}{*}{ 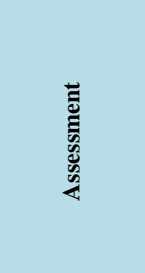 } & \multirow[b]{2}{*}{ 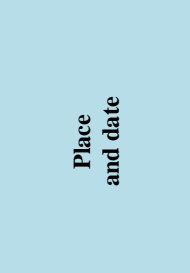 } & \multirow[b]{2}{*}{ 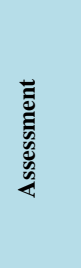 } & \multicolumn{2}{|c|}{$\tilde{\underline{z}}$} & \multirow[b]{2}{*}{ 氠 } \\
\hline & & & & & & & & & & 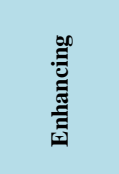 & 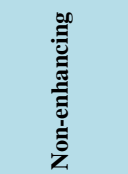 & \\
\hline 1 & $\begin{array}{c}\text { AMC, } \\
\text { March 10, } 1995 \\
\text { February 26, } \\
1999\end{array}$ & $\begin{array}{c}\text { A } \\
\text { GS }\end{array}$ & $\begin{array}{c}\text { AMC, } \\
\text { March 24, } 1999\end{array}$ & DIPG & $\begin{array}{l}\text { 2S 3RT 2C } \\
\text { HDC }\end{array}$ & $\begin{array}{l}\text { December 3, } \\
1999\end{array}$ & $\begin{array}{c}\text { Recurrent } \\
\text { tumor, DIPG } \\
\text { and } \\
\text { multicentric }\end{array}$ & $\begin{array}{c}\text { External } \\
\text { radiology, CRR, } \\
\text { April 12, } 2000\end{array}$ & CR & $\begin{array}{l}\text { Decreased } \\
\text { over } 50 \%\end{array}$ & Decreased R & esolved \\
\hline 2 & & & $\begin{array}{c}\text { AMC, } \\
\text { August 9, } 1999\end{array}$ & DIPG & $\mathrm{RT}$ & $\begin{array}{c}\text { November 3, } \\
1999\end{array}$ & $\begin{array}{l}\text { Recurrent } \\
\text { tumor }\end{array}$ & $\begin{array}{c}\text { External } \\
\text { radiology, CRR } \\
\text { February 23, } \\
2000\end{array}$ & PR & $\begin{array}{l}\text { Decreased } \\
\text { over } 50 \%\end{array}$ & Decreased & \\
\hline 3 & & & $\begin{array}{c}\text { AMC, } \\
\text { January 4, } 1999\end{array}$ & DIPG & RT C & April 27, 1999 & $\begin{array}{l}\text { Recurrent } \\
\text { tumor }\end{array}$ & $\begin{array}{c}\text { External } \\
\text { radiology, CRR } \\
\text { June 18, } 1999\end{array}$ & PR & $\begin{array}{l}\text { Decreased } \\
\text { over } 50 \%\end{array}$ & Decreased & \\
\hline 4 & & & $\begin{array}{c}\text { AMC, } \\
\text { April 11, } 2011\end{array}$ & DIPG & $\begin{array}{c}\text { RT C } \\
\text { Targeted } \\
\text { (bevacizumab) }\end{array}$ & $\begin{array}{c}\text { October 20, } \\
2011\end{array}$ & $\begin{array}{l}\text { Recurrent } \\
\text { tumor }\end{array}$ & $\begin{array}{l}\text { External } \\
\text { radiology, July } \\
30,2012\end{array}$ & PR & $\begin{array}{l}\text { Decreased } \\
\text { over } 50 \%\end{array}$ & Decreased & \\
\hline 5 & & & $\begin{array}{c}\text { RG, } \\
\text { December 3, } \\
1995\end{array}$ & DIPG & RT & $\begin{array}{c}\text { February 21, } \\
1996\end{array}$ & $\begin{array}{l}\text { Recurrent } \\
\text { tumor }\end{array}$ & $\begin{array}{l}\text { External } \\
\text { radiology, May } \\
\text { 22, } 1996\end{array}$ & SD & Stable & Stable & \\
\hline 6 & & & $\begin{array}{c}\text { AMC, } \\
\text { July } 31,1998\end{array}$ & DIPG & RT & $\begin{array}{l}\text { October 26, } \\
1998\end{array}$ & $\begin{array}{l}\text { Recurrent } \\
\text { tumor }\end{array}$ & $\begin{array}{c}\text { External } \\
\text { radiology, March } \\
18,1999\end{array}$ & SD & Stable & Stable & \\
\hline 7 & $\begin{array}{c}\text { AMC, } \\
\text { March 30, } 2005\end{array}$ & A2 & $\begin{array}{c}\text { RG, } \\
\text { March 10, } 2005\end{array}$ & DIPG & $\mathrm{C}$ & May 24, 2005 & $\begin{array}{l}\text { Recurrent } \\
\text { tumor }\end{array}$ & $\begin{array}{l}\text { External } \\
\text { radiology, } \\
\text { September 12, } \\
2005\end{array}$ & SD & Stable & Stable & \\
\hline 8 & & & $\begin{array}{c}\text { RG, } \\
\text { March 1, } 2010\end{array}$ & DIPG & RT C & July 27, 2010 & $\begin{array}{l}\text { Persistent } \\
\text { tumor }\end{array}$ & $\begin{array}{c}\text { RG, } \\
\text { May 26, } 2011\end{array}$ & SD & Stable & Stable & \\
\hline 9 & $\begin{array}{c}\text { AMC, } \\
\text { August 24, } \\
1999\end{array}$ & AA & $\begin{array}{c}\text { AMC, } \\
\text { August 20, } 1999\end{array}$ & $\begin{array}{l}\text { Right } \\
\text { thalamic } \\
\text { tumor }\end{array}$ & $\mathrm{RT}$ & $\begin{array}{c}\text { October 27, } \\
1999\end{array}$ & $\begin{array}{l}\text { Recurrent } \\
\text { tumor }\end{array}$ & $\begin{array}{l}\text { External } \\
\text { radiology, CRR, } \\
\text { April 12, } 2000\end{array}$ & PR & $\begin{array}{c}\text { Decreased } \\
\text { over } 50 \%\end{array}$ & Stable & \\
\hline 10 & $\begin{array}{c}\text { AMC, } \\
\text { December 24, } \\
1998\end{array}$ & AA & $\begin{array}{c}\text { AMC, } \\
\text { December 19, } \\
1997\end{array}$ & $\begin{array}{l}\text { Left } \\
\text { temporal } \\
\text { tumor }\end{array}$ & 2S RT 4C & $\begin{array}{c}\text { September 23, } \\
1999\end{array}$ & $\begin{array}{l}\text { Recurrent } \\
\text { tumor }\end{array}$ & $\begin{array}{c}\text { External } \\
\text { radiology, } \\
\text { June } 18,2000\end{array}$ & SD & Stable & Stable & \\
\hline 11 & $\begin{array}{c}\text { AMC, } \\
\text { May 19, } 2003\end{array}$ & GBM & $\begin{array}{c}\text { AMC, } \\
\text { May 12, } 2003\end{array}$ & $\begin{array}{l}\text { Left } \\
\text { temporal } \\
\text { tumor }\end{array}$ & S RT C & August 20, 2003 & $\begin{array}{l}\text { Recurrent } \\
\text { tumor }\end{array}$ & $\begin{array}{c}\text { External } \\
\text { radiology, } \\
\text { December 4, } 2003\end{array}$ & SD & Stable I & Decreased & \\
\hline 12 & $\begin{array}{c}\text { AMC, } \\
\text { May 8, } 2009\end{array}$ & GBM & $\begin{array}{c}\text { AMC, } \\
\text { June 2, } 2009\end{array}$ & $\begin{array}{l}\text { Right } \\
\text { temporal } \\
\text { tumor }\end{array}$ & 2S RT 2C & $\begin{array}{l}\text { October 14, } \\
2009\end{array}$ & $\begin{array}{l}\text { Recurrent } \\
\text { tumor }\end{array}$ & $\begin{array}{c}\text { External } \\
\text { radiology, } \\
\text { February 8, } 2010\end{array}$ & SD & Stable & Stable & \\
\hline
\end{tabular}

*This table includes patients whose responses were determined as CR, PR and SD only, which are of main interest to the readers. Radiology diagnosis is different than pathology diagnosis because the radiology diagnosis is based on the location of the tumor in the brain and the pathology diagnosis is based on the microscopic appearance. Abbreviations: A-astrocytoma; A2-astrocytoma, grade 2; AA-anaplastic astrocytoma; AMC-academic medical center; Cchemotherapy; CR — complete response; CRR—central radiology review; DIPG—diffuse intrinsic pontine glioma; GBM-glioblastoma multiforme; GS — gliosarcoma; HDC—high-dose chemotherapy; PR—partial response; RG—regional hospital; RT—radiation therapy; S—surgery; SD—stable disease.

\section{Safety and Adverse Events}

\section{Study Population}

Safety assessments were analyzed based upon the total number of enrolled patients in the study $(n=43)$. Intense monitoring of patient safety was conducted during the first two months of therapy and involved daily direct 


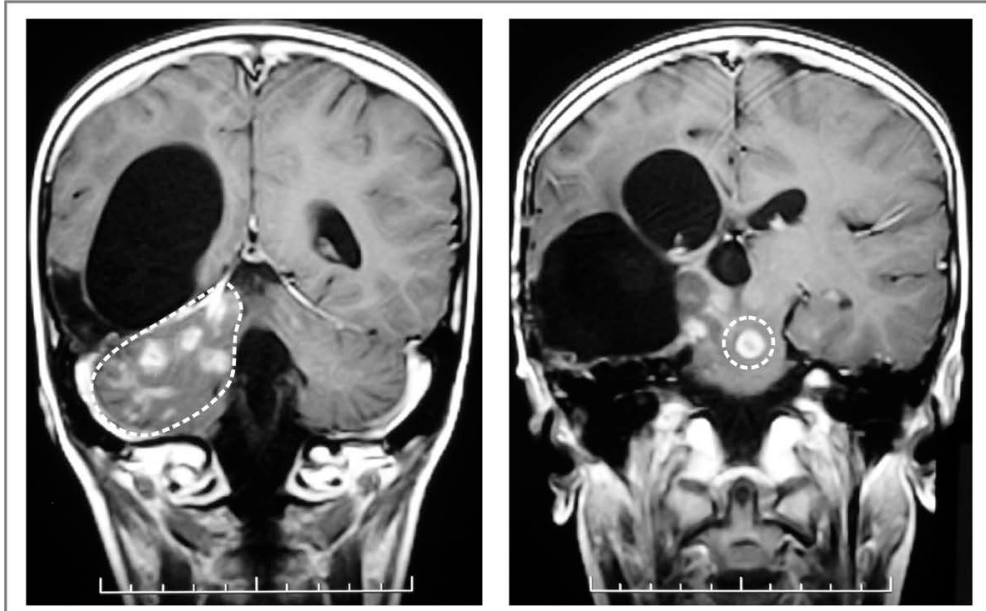

A. Baseline - Dec. 03, 1999

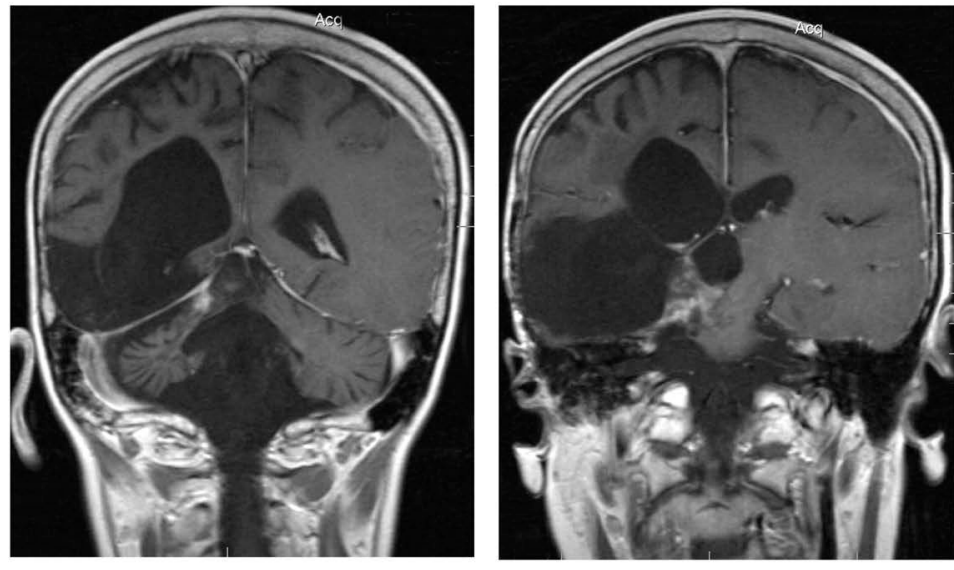

B. June 26,2006

Figure 1. Patient 1. (A) Baseline MRI; (B) Follow-up MRI indicating PR. Coronal T-1 contrast-enhanced images. A CR was confirmed by PET scan.

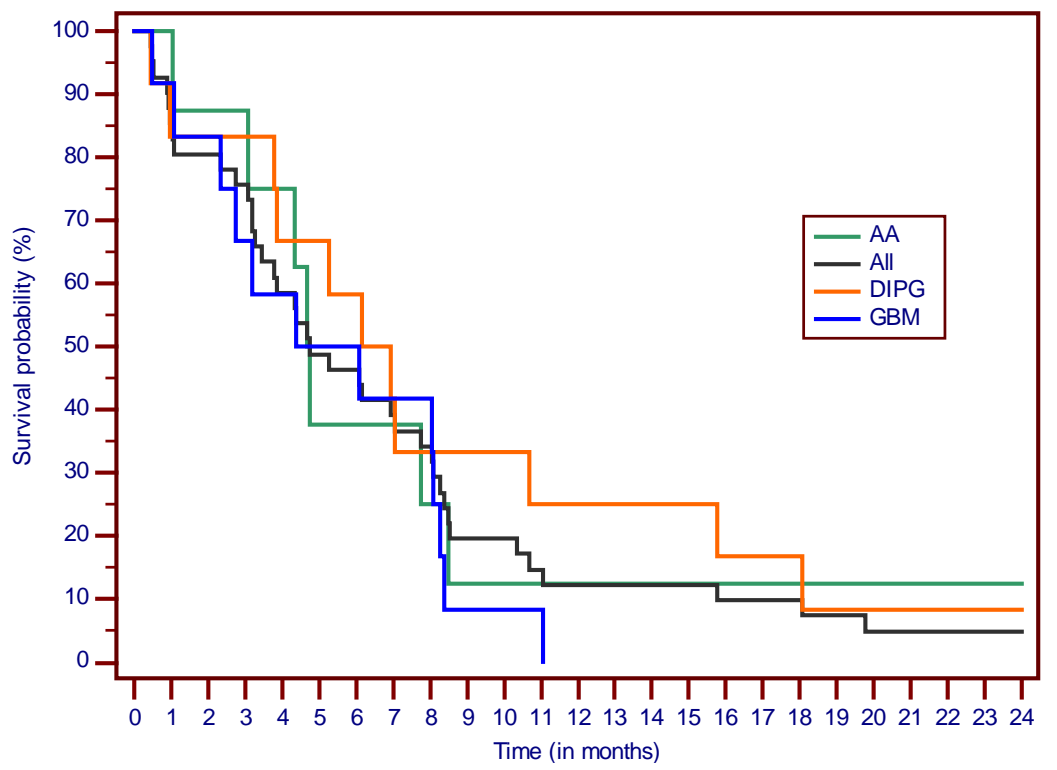

Figure 2. The Kaplan-Meier survival curve. Overall survival from the treatment start. 


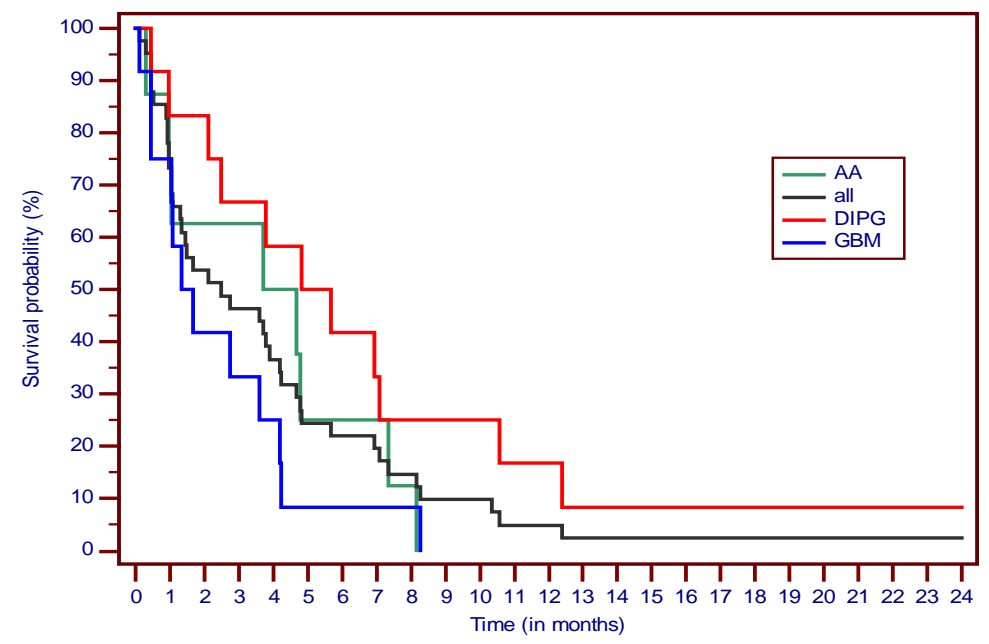

Figure 3. The Kaplan-Meier survival curve. Progression-free survival from the treatment start.

Table 4. Response rates and overall survival.

\begin{tabular}{|c|c|c|c|c|c|c|c|}
\hline \multirow{2}{*}{ Diagnosis } & \multirow[b]{2}{*}{$\mathbf{N}$} & \multicolumn{5}{|c|}{ Response N (\%) } & \multirow{2}{*}{$\begin{array}{c}\text { Survival } \\
\begin{array}{c}\text { Median (range) } \\
\text { in months }\end{array}\end{array}$} \\
\hline & & CR & PR & SD & PD & NE & \\
\hline DIPG & 12 & 1 & 3 & 4 & 2 & 2 & $6.1\left(0.5-173.5^{+}\right)$ \\
\hline GBM & 12 & & & 2 & 8 & 2 & $4.4(0.5-11.1)$ \\
\hline AA & 8 & & 1 & 1 & 3 & 3 & $4.7(1.1-136.7)$ \\
\hline Medulloblastoma & 3 & & & & & 3 & $0.9(0.5-0.9)$ \\
\hline sPNET & 3 & & & & 3 & & $3.4(3.3-8.5)$ \\
\hline PAD & 1 & & & & 1 & & 19.8 \\
\hline $\mathbf{A E}$ & 1 & & & & & 1 & 10.5 \\
\hline AT/RT & 1 & & & & 1 & & 3.2 \\
\hline TOTAL & 41 & $1(2.4)$ & $4(9.8)$ & 7 (17.1) & 18 (43.9) & $11(26.8)$ & $4.8(0.5-173.5+)$ \\
\hline
\end{tabular}

Abbreviations: AA —anaplastic astrocytoma; AE_anaplastic ependymoma; AT/RT—atypical teratoid rhabdoid tumor; CR—complete response; DIPG—diffuse intrinsic brainstem glioma; GBM—glioblastoma multiforme; NE—non-evaluable; PAD—disseminated pilocytic astrocytoma; PD—progressive disease; PR—partial response; SD—stable disease; sPNET—supratentorial primitive neuroectodermal tumor. +Patient alive.

questioning concerning adverse events, initially at the clinic and then followed by daily phone calls during the home administration phase. After two months, telephone contact was conducted on at least a weekly basis. Adverse events were coded and graded according to CTCAE v.3. No long-term adverse drug events (ADEs) to ANPs were reported.

ADEs grades 3 and 4 identified and reported by study patients in comparison to other studies is shown in Table 6.

Brain tumor patients frequently receive corticosteroids as part of their therapeutic regimen to reduce cerebral edema around tumors. The use of corticosteroids, the infusion of large volumes of sodium-containing solutions during ANP therapy, and the brain tumor itself predispose a patient to an increased incidence of serum sodium concentration abnormalities. As a result, grade 3 reversible hypernatremia was reported in two cases and grade 4 hypernatremia was reported in six cases ( $4.6 \%$ and $13.9 \%$ respectively). In the studies with temozolomide, there was substantial myelotoxicity, vomiting, and incidence of infection including sepsis and pneumonia reported in Table 6. 
Table 5. Phase II clinical studies in recurrent or progressive brain tumors in children. The results of chemotherapy with temozolomide vs. antineoplastons.

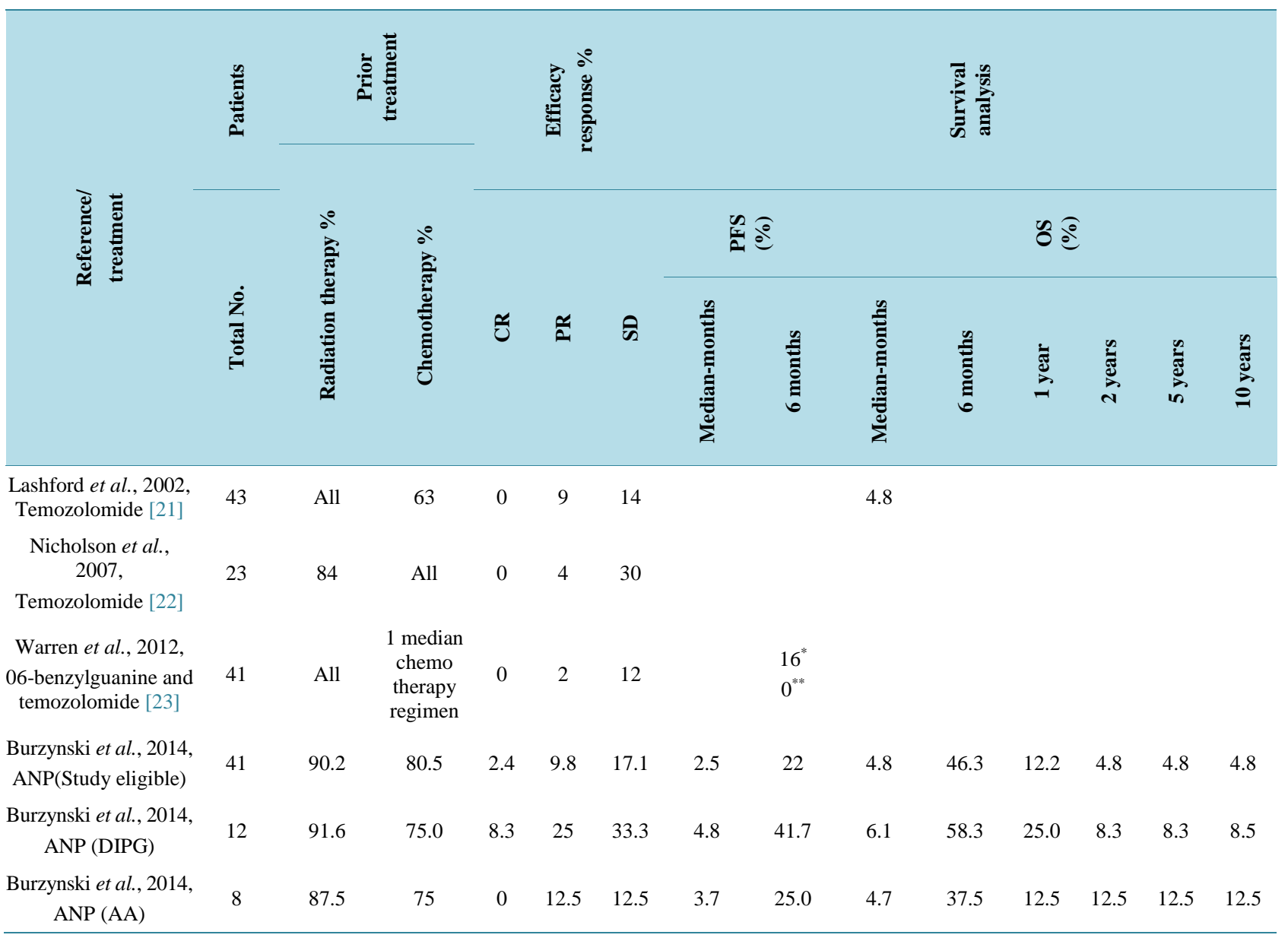

Abbreviations: AA — anaplastic astrocytoma; ANP — antineoplastons A10 and AS2-1; CR—complete response; DIPG—diffuse intrinsic pontine glioma; HGG—-high grade glioma; OS—overall survival; PFS—progression-free survival; PR—partial response; SD—stable disease; ${ }^{*}$ Stratum A, HGG; ${ }^{* *}$ Stratum B, DIPG.

\section{Discussion}

The breakthrough in molecular and genomic studies brought justified excitement into the research on primary malignant brain tumors [20]. Until these new data are translated into clinical results it is necessary to rely on currently available treatment modalities which do not prove the efficacy beyond standard RT, which remains the accepted treatment for newly-diagnosed HGG and DIPG. Clinical trials in pediatric HGG and DIPG were extensively reviewed previously [6] [7] [9]. Recently, seven trials were conducted for a mixed population of pediatric patients, usually for HGG with a percentage of patients diagnosed with DIPG. These studies were reviewed by us in recent publications [10] [12]. Lashford et al., Nicholson et al. and Warren et al. reported on studies with temozolomide in the treatment of children diagnosed with HGG and DIPG [21]-[23]. In these studies, 23 to 43 children who failed radiation therapy and chemotherapy were recruited. No CRs in their studies were accomplished, but they reported from $2 \%$ to $9 \%$ for PR and $14 \%$ to $30 \%$ for SD. Limited survival data were reported. There were no median PFSs determined and 6 months PFS in the Warren et al. study was 16\% for HGG and 0\% for DIPG [23]. OS was only reported by Lashford et al. as 4.8 months median survival (Table 5).

The results of our clinical trials indicate a significant percentage of CR and PR in a small group of recurrent DIPG (4 out of 12 patients). In the recurrent AA group of eight patients, there were single cases of PR and SD. It should be emphasized that the study accrued a very difficult-to-treat population compared to other ANP studies provided rationale for future Phase III studies. 
Table 6. Incidence of adverse drug experiences (ADEs), grades 3 and 4, in antineoplastons study (ITT) compared to selected studies with temozolomide for pediatric brain tumors.

\begin{tabular}{|c|c|c|c|c|c|}
\hline \multirow[t]{2}{*}{$\begin{array}{c}\text { ADE } \\
\text { (Incidence \%) }\end{array}$} & \multicolumn{2}{|c|}{ 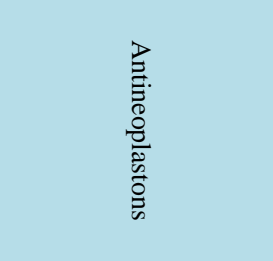 } & \multirow{2}{*}{ 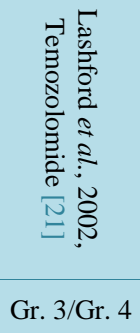 } & \multicolumn{2}{|c|}{ 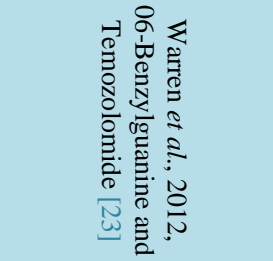 } \\
\hline & Gr. 3 & Gr. 4 & & Gr. 3 & Gr. 4 \\
\hline Hypokalemia & & 2.3 & & & \\
\hline Hypernatremia & 4.6 & 13.9 & & & \\
\hline Fatigue & & & 1 & & \\
\hline Somnolence & 2.3 & 2.3 & & & \\
\hline Thrombocytopenia & & & 7 & 17 & 2 \\
\hline Neutropenia & & & & 12 & 32 \\
\hline Leukopenia & & & 5 & 15 & 10 \\
\hline Lymphopenia & & & & 24 & 2 \\
\hline Anemia & & & & 7 & \\
\hline Vomiting & & & 2 & 2 & \\
\hline Anorexia & & & $<1$ & & \\
\hline Infection & & & & 5 & \\
\hline Sepsis & & & 2 & & \\
\hline Pneumonia & & & 2 & & \\
\hline \multicolumn{6}{|l|}{ Fever } \\
\hline Neuropathy & & & $<1$ & & \\
\hline Seizures & & & $<1$ & & \\
\hline Headache & & & & 2 & \\
\hline Pain & & & $<1$ & & \\
\hline
\end{tabular}

\section{Conclusion}

The treatment of recurrent primary malignant pediatric brain tumors creates great challenge for neuro-oncologists. This Phase II study of Antineoplastons A10 and AS2-1 reported encouraging responses and survival data in the heavily pretreated and refractory patient population. Of special interest are the results in recurrent DIPG and AA. The study reached the goal of four or more cases of OR in the ITT population. The data acquired in patients with recurrent DIPG and AA present improved survival outcomes due to ANP therapy and tolerable toxicity. The additional Phase II studies of ANP in pediatric tumors and currently organized Phase III studies in newly-diagnosed DIPG will provide additional data.

\section{Acknowledgements}

The authors express their appreciation to the doctors involved in the care of the patients: Drs. Alejandro Marquis, Robert A. Weaver, Robert I. Lewy, Eva Kubove, Barbara Szymkowski, and Mohammad Khan. Preparation of the manuscript was provided by Carolyn Powers, Adam Golunski, and Jennifer Pineda. 


\section{Conflict of Interest}

The clinical trial was sponsored by Burzynski Research Institute, Inc. The authors are employed by Burzynski Clinic. Dr. Stanislaw R. Burzynski and Dr. Gregory S. Burzynski are share holders in Burzynski Research Institute, Inc. Dr. Stanislaw R. Burzynski is President of Burzynski Research Institute, Inc., Dr. Tomasz Janicki is Vice President of Clinical Trials at Burzynski Research Institute, Inc., Dr. Gregory S. Burzynski is Vice President of Burzynski Clinic, and Dr. Sheldon Brookman is Director of Pharmaceutical Development at Burzynski Clinic.

\section{References}

[1] Porter, K.R., McCarthy, B.J., Freels, S., et al. (2010) Prevalence Estimates for Primary Brain Tumors in the United States by Age, Gender, Behavior and Histology. Neuro-Oncology, 12, 520-527. http://dx.doi.org/10.1093/neuonc/nop066

[2] CBTRUS (2013) CBTRUS Statistical Report Supplement 2013: Primary Brain and Central Nervous System Tumors Diagnosed in the United States in 2006-2010. Neuro-Oncology, 15, ii1-ii56.

[3] Broniscer, A. and Gajjar, A. (2004) Supratentorial High-Grade Astrocytoma and Diffuse Brainstem Glioma: Two Challenges for the Pediatric Oncologist. Oncologist, 9, 197-206. http://dx.doi.org/10.1634/theoncologist.9-2-197

[4] Stupp, R., Mason, W.P., van den Bent, M.J., Weller, M., Fisher, B., et al. (2005) Radiotherapy plus Concomitant and Adjuvant Temozolomide for Glioblastoma. The New England Journal of Medicine, 352, 987-996. http://dx.doi.org/10.1056/NEJMoa043330

[5] Wick, W., Weller, M., Weiler, M., Batchelor, T., Yung, A.W. and Platten, M. (2011) Pathway Inhibition: Emerging Molecular Targets for Treating Glioblastoma. Neuro-Oncology, 13, 566-579. http://dx.doi.org/10.1093/neuonc/nor039

[6] Hargrave, D., Bartels, U. and Bouffet, E. (2006) Diffuse Brainstem Glioma in Children: Critical Review of Clinical Trials. Lancet, 7, 241-248.

[7] Finlay, J.L. and Zacharoulis, S. (2005) The Treatment of High Grade Gliomas and Diffuse Intrinsic Pontine Tumors of Childhood and Adolescence: A Historical and Futuristic Perspective. Journal of Neuro-Oncology, 75, 253-266. http://dx.doi.org/10.1007/s11060-005-6747-7

[8] Burzynski, S.R. (2004) The Present State of Antineoplaston Research (1). Integrative Cancer Therapies, 3, 47-58. http://dx.doi.org/10.1177/1534735403261964

[9] Burzynski, S.R. (2006) Treatments for Astrocytic Tumors in Children: Current and Emerging Strategies. Pediatric Drugs, 8, 167-168. http://dx.doi.org/10.2165/00148581-200608030-00003

[10] Burzynski, S.R., Janicki, T.J., Burzynski, G.S. and Marszalek, A. (2014) The Response and Survival of Children with Recurrent Diffuse Intrinsic Pontine Gliomas Based on Phase II Study of Antineoplastons A10 and AS2-1 in Patients with Brainstem Glioma. Child's Nervous System, Published Online.

[11] Burzynski, S.R., Janicki, T.J., Burzynski, G.S. and Marszalek, A. (2014) Long-Term Survival (>13 Years) in a Child with Recurrent Diffuse Intrinsic Pontine Glioma: A Case Report. Journal of Pediatric Hematology/Oncology, Published Oniline.

[12] Burzynski, S.R., Janicki, T.J., Burzynski, G.S. and Marszalek, A. (2014) A Phase II Study of Antineoplastons A10 and AS2-1 in Children with High-Grade Glioma. Final Report (Protocol BT-06), and Review of Recent Trials. Journal of Cancer Therapy, 5, 565-577. http://dx.doi.org/10.4236/jct.2014.56065

[13] Packer, R.J., Boyett, J.M., Zimmerman, R.A., Rorke, L.B., Kaplan, A.M., Albright, A.L., et al. (1993) Hyperfractionated Radiation Therapy (72 Gy) for Children with Brain Stem Gliomas. A Childrens Cancer Group Phase I/II Trial. Cancer, 72, 1414-1421.

[14] Albright, A.L., Packer, R.J., Zimmerman, R., Rorke, L.B., Boyett, J. and Hammond, G.D. (1993) Magnetic Resonance Scans Should Replace Biopsies for the Diagnosis of Diffuse Brain Stem Gliomas: A Report from the Children's Cancer Group. Neurosurgery, 33, 1026-1030. http://dx.doi.org/10.1227/00006123-199312000-00010

[15] Wen, P.Y., Macdonald, D.R., Reardon, D.A., Cloughesy, T.F., Sorensen, A.G., Galanis, E., et al. (2010) Updated Response Assessment Criteria for High-Grade Gliomas: Response Assessment in Neuro-Oncology Working Group. Journal of Clinical Oncology, 28, 1963-1972. http://dx.doi.org/10.1200/JCO.2009.26.3541

[16] Choux, M., Lena, G. and Do, L. (2000) Brainstem Tumors. In: Choux, M., Di Rocco, C. and Hockley, A., Eds., Pediatric Neurosurgery, Churchill Livingstone, New York, 471-491.

[17] Leach, P.A., Estlin, E.J., Coope, D.J., Thorne, J.A. and Kamaly-Asl, I.D. (2008) Diffuse Brainstem Gliomas in Children: Should We or Shouldn't We Biopsy? British Journal of Neurosurgery, 22, 619-625. http://dx.doi.org/10.1080/02688690802366198 
[18] Chang, S.M., Kuhn, J.G., Robins, H.I., Schold, S.C., Spence, A.M., Berger, M.S., et al. (1999) Phase II Study of Phenylacetate in Patients with Recurrent Malignant Glioma: A North American Brain Tumor Consortium Report. Journal of Clinical Oncology, 17, 984-990.

[19] Weller, M., Cloughesy, T., Perry, J.R. and Wisk, W. (2013) Standards of Care for Treatment of Recurrent Glioblastoma-Are We There Yet? Neuro-Oncology, 15, 4-27. http://dx.doi.org/10.1093/neuonc/nos273

[20] Van Meir, E.G., Hadjipanayis, C.G., Norden, A.D., Shu, H.K., Wen, P.Y. and Olson, J.J. (2010) Exciting New Advances in Neuro-Oncology: The Avenue to a Cure for Malignant Glioma. CA: A Cancer Journal for Clinicians, 60, 166-193. http://dx.doi.org/10.3322/caac.20069

[21] Lashford, L.S., Thiesse, P., Jouvet, A., Jaspan, T., Couanet, D., Griffiths, P.D., et al. (2002) Temozolomide in Malignant Gliomas of Childhood: A United Kingdom Children's Cancer Study Group and French Society for Pediatric Oncology Intergroup Study. Journal of Clinical Oncology, 20, 4684-4691. http://dx.doi.org/10.1200/JCO.2002.08.141

[22] Nicholson, H.S., Kretschmar, C.S., Krailo, M., Bernstein, M., Kadota, R., Fort, D., et al. (2007) Phase 2 Study of Temozolomide in Children and Adolescents with Recurrent Central Nervous System Tumors. A Report from the Children's Oncology Group. Cancer, 110, 1542-1550. http://dx.doi.org/10.1002/cncr.22961

[23] Warren, K.E., Gururangan, S., Geyer, J.R., McLendon, R.E., Poussaint, T.Y., Wallace, D., et al. (2012) A Phase II Study of 06-Benzylguanine and Temozolomide in Pediatric Patients with Recurrent or Progressive High-Grade Gliomas and Brainstem Gliomas: A Pediatric Brain Tumor Consortium Study. Journal of Neuro-Oncology, 106, 643-649. http://dx.doi.org/10.1007/s11060-011-0709-z 
Scientific Research Publishing (SCIRP) is one of the largest Open Access journal publishers. It is currently publishing more than 200 open access, online, peer-reviewed journals covering a wide range of academic disciplines. SCIRP serves the worldwide academic communities and contributes to the progress and application of science with its publication.

Other selected journals from SCIRP are listed as below. Submit your manuscript to us via either submit@scirp.org or Online Submission Portal.
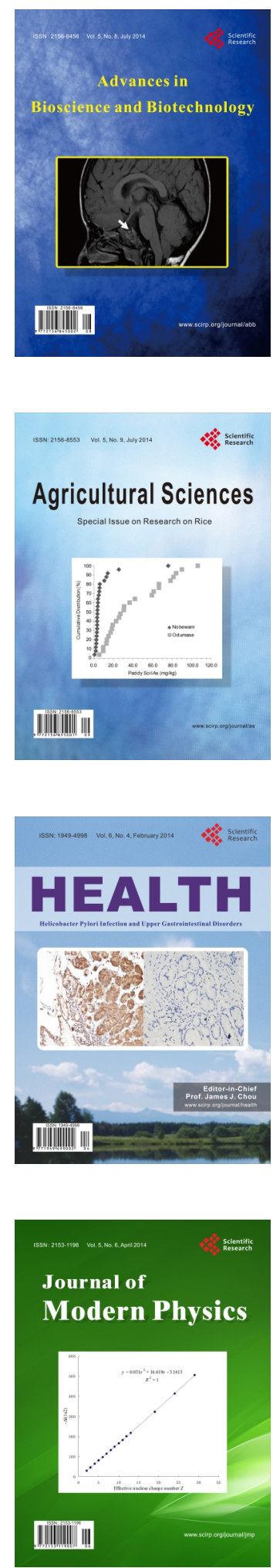
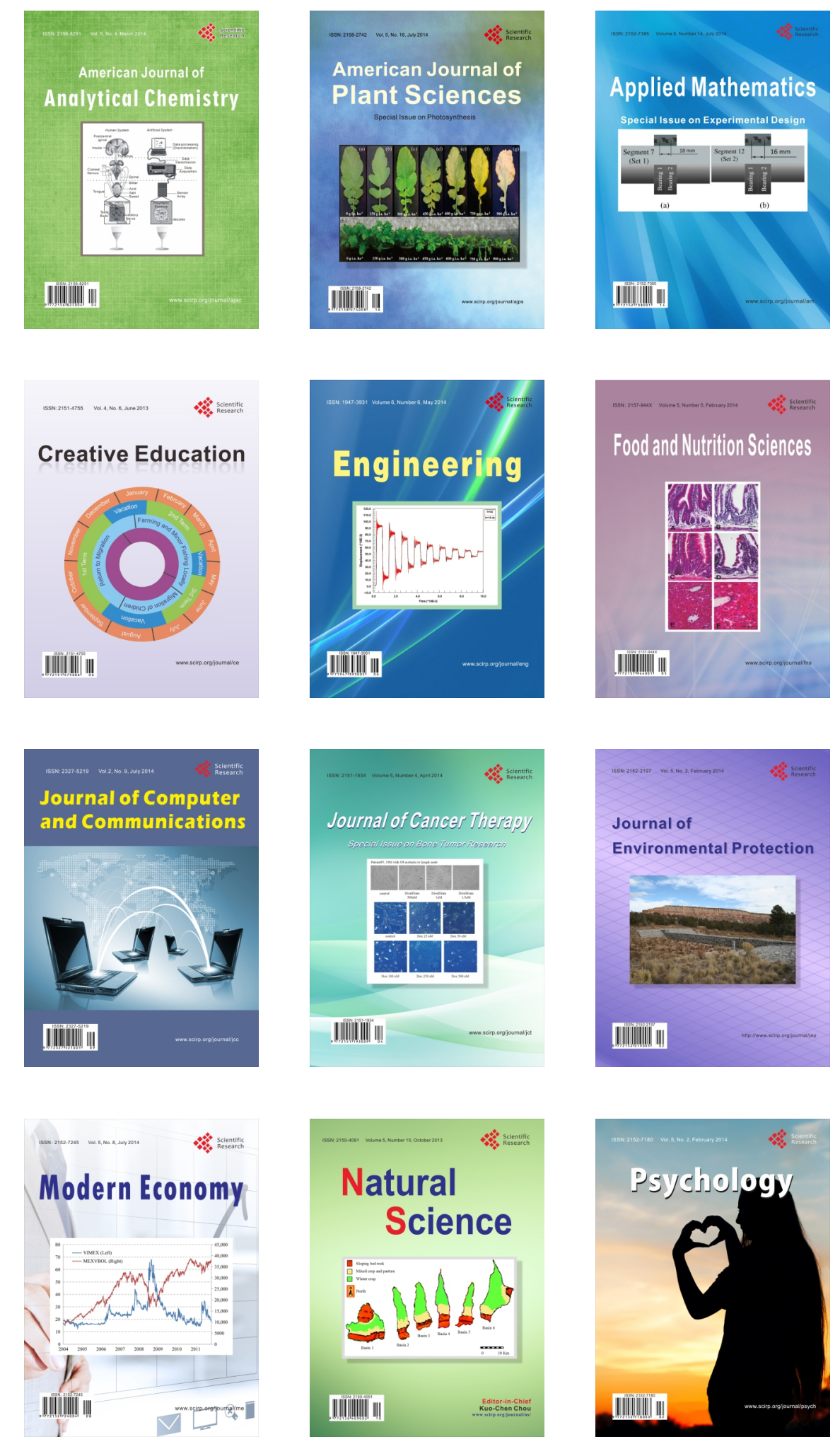